\title{
BMJ Open Modification of diet, exercise and lifestyle (MODEL) study: a randomised controlled trial protocol
}

\author{
Simone Radavelli-Bagatini (D) , , Catherine P Bondonno, ${ }^{1,2}$ Marc Sim (D) ,1,2 \\ Lauren C Blekkenhorst, ${ }^{1,2}$ Reindolf Anokye, ${ }^{1}$ Emma Connolly, ${ }^{1}$ \\ Nicola P Bondonno, ${ }^{1,2}$ John T Schousboe, ${ }^{3}$ Richard J Woodman, ${ }^{4}$ Kun Zhu, ${ }^{2,5}$ \\ Pawel Szulc, ${ }^{6}$ Ben Jackson, ${ }^{7}$ James Dimmock, ${ }^{8}$ Markus P Schlaich, ${ }^{2}$ Kay L Cox, ${ }^{2}$ \\ Douglas P Kiel, ${ }^{9}$ Wai H Lim, ${ }^{2,10}$ Mandy Stanley (D) , ${ }^{1}$ Amanda Devine, \\ Peter L Thompson, ${ }^{11}$ Jenny Gianoudis, ${ }^{12}$ Belinda De Ross, ${ }^{12}$ Robin M Daly, ${ }^{12}$ \\ Joshua R Lewis, ${ }^{1,2,13}$ Jonathan M Hodgson ${ }^{1,2}$
}

To cite: Radavelli-Bagatini S, Bondonno CP, Sim M, et al. Modification of diet, exercise and lifestyle (MODEL) study: a randomised controlled trial protocol. BMJ Open 2020;10:e036366. doi:10.1136/ bmjopen-2019-036366

- Prepublication history for this paper is available online. To view these files, please visit the journal online (http://dx.doi. org/10.1136/bmjopen-2019036366).

RMD, JRL and JMH contributed equally.

Received 12 December 2019 Revised 18 March 2020 Accepted 18 May 2020

Check for updates

(C) Author(s) (or their employer(s)) 2020. Re-use permitted under CC BY-NC. No commercial re-use. See rights and permissions. Published by BMJ.

For numbered affiliations see end of article.

Correspondence to

Dr Simone Radavelli-Bagatini; simoneradavelli@hotmail.com

\section{ABSTRACT}

Introduction Most cardiovascular disease (CVD)-related events could be prevented or substantially delayed with improved diet and lifestyle. Providing information on structural vascular disease may improve CVD risk factor management, but its impact on lifestyle change remains unclear. This study aims to determine whether providing visualisation and pictorial representation of structural vascular disease (abdominal aortic calcification (AAC)) can result in healthful diet and lifestyle change.

Methods and analysis This study, including men and women aged 60-80 years, is a 12-week, two-arm, multisite randomised controlled trial. At baseline, all participants will have AAC assessed from a lateral spine image captured using a bone densitometer. Participants will then be randomised to receive their AAC results at baseline (intervention group) or a usual care control group that will receive their results at 12 weeks. All participants will receive information about routinely assessed CVD risk factors and standardised (video) diet and lifestyle advice with three simple goals: (1) increase fruit and vegetable (FV) intake by at least one serve per day, (2) improve other aspects of the diet and (3) reduce sitting time and increase physical activity. Clinical assessments will be performed at baseline and 12 weeks.

Outcomes The primary outcome is a change in serum carotenoid concentrations as an objective measure of FV intake. The study design, procedures and treatment of data will adhere to Standard Protocol Items for Randomized Trials guidelines.

Ethics and dissemination Ethics approval for this study has been granted by the Edith Cowan University and the Deakin University Human Research Ethics Committees (Project Numbers: 20513 HODGSON and 2019-220, respectively). Results of this study will be published in peer-reviewed academic journals and presented in scientific meetings and conferences. Information regarding consent, confidentiality, access to data, ancillary and posttrial care and dissemination policy has been disclosed in the participant information form.

Trial registration number Australian New Zealand Clinical Trial Registry (ACTRN12618001087246).

\section{Strengths and limitations of this study}

To date, no studies have investigated whether providing visualisation and pictorial representation of structural vascular disease in the abdominal aorta (abdominal aortic calcification (AAC)) assessed on scans from bone densitometers can result in greater uptake and adherence to dietary and physical activity recommendations.

- AAC from widely available bone densitometers is a quick, cost-effective and reproducible method to visualise structural vascular disease.

- Given the advantages of the imaging modality, this test may be a useful tool to encourage individuals to make healthful lifestyle choices and general practitioners to reinforce healthful lifestyle recommendations and encourage initiation and adherence to cardiovascular medications.

- A limitation is that this is a short-term 12-week randomised controlled trial and as such we cannot determine long-term dietary and lifestyle differences between the control and intervention arms.

\section{INTRODUCTION}

Background and rationale

Cardiovascular diseases (CVDs) are the leading cause of death. Globally, over $30 \%$ of all deaths are attributable to CVDs, with the majority of these due to either ischemic heart disease (heart attack) or cerebrovascular disease (stroke). ${ }^{1}$ In Australia, approximately $29 \%$ of all deaths have an underlying cause of CVD. ${ }^{2}$ Often heart attacks and strokes are the first clinical symptom of CVD with approximately $31 \%$ of first events being fatal. ${ }^{3}$ Furthermore, most survivors have substantial reductions in quality of life (QoL) due to changes in functional capacity.

Poor lifestyle behaviours contribute to the majority of CVD globally; improving 
diet, increasing physical activity and the cessation of smoking can substantially reduce the burden of CVD. ${ }^{4}$ Even modest sustained lifestyle changes, such as adding an extra serve of fruit and vegetable (FV) to the diet or reducing sitting time, can lower CVD risk. ${ }^{5}$ Growing evidence shows that a diet rich in FV (eg, two and five daily serves of fruit and vegetable, respectively), can lower the risk of CVD death, non-cardiovascular death and death from all causes. ${ }^{6-9}$

Worldwide, over 5 million deaths per year are attributed to diets low in $\mathrm{FV}^{7}$ well over 10 million deaths per year are attributed to lifestyle, including poor diet, physical inactivity and smoking. Although there is an estimated $20 \%$ lower risk of CVD comparing high to low FV intake (a $5 \%$ lower risk for each additional serving), ${ }^{6}$ most adults do not meet the minimum recommended servings for fruits (2 servings; $\geq 300 \mathrm{~g} /$ day) or vegetables ( $~ 5$ servings; $\geq 375 \mathrm{~g} /$ day) ${ }^{1011} \mathrm{In}$ addition, $70 \%-80 \%$ of adults fail to engage in the recommended amount of physical activity. ${ }^{12}$ Improved diet and increased physical activity would reduce the health expenditure associated with chronic diseases. ${ }^{1314}$

Current policy to prevent chronic disease is predicated on detection of the traditional, recognised risk factors such as altered blood lipids and elevated blood pressure (BP). However, new non-invasive tests that strongly predict disease presence, development, progression and outcome may lead to a paradigm shift in CVD risk management and primary prevention of clinical disease. ${ }^{15}$ Vascular calcification can be used to assess atherosclerotic vascular disease presence and burden. It can be measured by $\mathrm{CT}$ in the coronary arteries (coronary artery calcification (CAC)) and X-ray in the abdominal aorta (abdominal aortic calcification (AAC)). The presence of any CAC or any AAC provides a measurement of the amount of calcium deposited in arteries, which are the surrogates for atherosclerosis, strongly predict CVD events and substantially improve both sensitivity and specificity to the identification of future CVD events. ${ }^{16-18}$ Furthermore, new calcified lesions (progression) are evidence of active atherosclerosis likely to be related to poorer patient outcomes. ${ }^{19}$ These methods represent a significant advancement in CVD risk estimation and may be used in primary prevention. However, due to cost and radiation concerns, these tests are not used for community-based or routine screening. In contrast, AAC assessed from lateral spine images used to detect vertebral fractures from dual energy X-ray absorptiometry (DXA) scans, offer an alternative and widely available method which has ultra-low to negligible radiation $(0.005 \mathrm{mSv})$, low cost and, consequently, can be used routinely in almost all clinical populations.

Good evidence suggests that CAC screening improves medication adherence and CVD risk factor management. ${ }^{20}{ }^{21}$ However, there are limited data as to whether CAC screening can motivate individuals towards beneficial behavioural or lifestyle changes to improve CVD risk. ${ }^{21}$ To date, most of the available data in this area are mixed $^{21}$ and derived from observational studies, with only a few randomised controlled trials (RCTs) conducted. ${ }^{21}$

Providing individuals with visualisation and pictorial representation of structural vascular disease followed by diet and lifestyle advice could stimulate protective health behaviours. This approach is supported by wellestablished models of health promotion and preventive behaviour. ${ }^{22-24}$ To date, no studies have investigated whether providing individuals with visualisation and pictorial representation of the presence and severity of advanced structural vascular disease in the abdominal aorta can improve both diet and physical activity, the primary modifiable causes of CVD. In addition, AAC scanning is a relatively new technique compared with CAC scanning. It is important to examine within an RCT framework the extent to which this technique can be used as a tool to encourage healthy behaviour change and monitor progression of CVD.

\section{Objectives}

The primary aim of this study is to investigate, for the first time, whether providing individuals with visualisation and pictorial representation of structural vascular disease presence and severity, assessed by widely available bone densitometers, can increase objective measures of FV intake (serum carotenoids) after 12 weeks. Major secondary aims are to evaluate whether providing visualisation and pictorial representation of structural vascular disease will affect (1) adherence to other dietary recommendations (eg, sodium, fibre, whole grains, seeds and nuts); (2) adherence to physical activity and sedentary behaviour (eg, sitting time) recommendations; (2) CVD risk factors (such as BP, lipids and glucose levels) and; (4) other health-related measures (eg, gut health, physical function and mental health).

\section{Trial design}

This will be a 12-week, single-blind, two-arm, multisite RCT. The study trial complies with the Standard Protocol Items for Randomized Trials recommendations and guidelines for protocols. ${ }^{25}$ This study will run in parallel at the Royal Perth Hospital Medical Research Foundation, School of Medical and Health Sciences at Edith Cowan University (Perth site) and within the Institute for Physical Activity and Nutrition, School of Exercise and Nutrition Sciences, Melbourne Burwood Campus at Deakin University (Melbourne site). Participants will be randomised (1:1) to either be provided with their AAC results at baseline (intervention group) or delayed until 12 weeks at the end of the RCT (control group). Participants will be stratified according to the age (above or below 70 years) and sex. A detailed process evaluation will be performed to assess the suitability, reliability and implementation of all processes involved (Anokye R, Radavelli-Bagatini S, Bondonno CP, et al. Process evaluation protocol for a multicentre, single-blind randomized controlled trial: the MODEL study. Under review by the BMJ Open (2019)). 


\section{METHODS}

Participants, interventions and outcomes

Study settings

This study will be conducted in ambulant communitydwelling Australian men and women, aged $60-80$ years, recruited from the general population in metropolitan Perth and Melbourne and surrounding areas in Australia.

\section{Eligibility criteria}

A total of 200 participants $(n=100$ control group; $n=100$ intervention group) from Perth and Melbourne will be recruited from the general population via media advertisements and participant databases from previous studies. A two-step screening process will be performed. Eligibility will be assessed initially via a telephone prescreen, followed by clinical assessments. An initial telephone screening questionnaire will be used to determine the eligibility of potential participants based on the inclusion/exclusion criteria. Volunteers who are eligible and willing to proceed will be sent an information pack and a clinic assessment visit will be scheduled for further screening and testing.

\section{Inclusion and exclusion criteria}

Participants included will be ambulant men and women aged 60-80 years. Exclusion criteria are (1) Body mass index (BMI) $\geq 37 \mathrm{~kg} / \mathrm{m}^{2}$ or BMI $<18 \mathrm{~kg} / \mathrm{m}^{2}$ or body weight $>125 \mathrm{~kg}$; (2) type 1 diabetes; (3) type 2 diabetes if greater than 10 years since diagnosis; (4) history of CVD events including ischemic heart disease, cerebrovascular disease, cardiomyopathy and peripheral arterial disease; (5) prior CAC or coronary angiograms (within the last 2 years); (6) use of anticoagulant therapy (warfarin); (7) those with significant comorbidities, such as chronic kidney disease (estimated glomerular filtration rate (eGFR) $<30 \mathrm{~mL} /$ $\min / 1.73 \mathrm{~m}^{2}$ ), cancer (current) or heart failure (class 3 and 4$)^{26}$; (8) systolic BP $>160 \mathrm{~mm} \mathrm{Hg}$ or diastolic BP $>100 \mathrm{~mm} \mathrm{Hg}$; (9) poor-image quality of the DXA scans resulting in an inability to assess the AAC and (10) those unable or unwilling to follow the study protocol. Eligible participants will be asked to sign an informed consent form prior to participation.

\section{Assessments}

The study flow chart is summarised in figure 1. At baseline, all participants will attend their screening appointment and baseline assessments, which includes their AAC assessment from a lateral spine image captured using a DXA densitometer. Participants will be then randomised to either the control or the intervention group. Although all participants will have their AAC scan performed at baseline, only the intervention group will be provided visualisation and pictorial representation of their AAC at baseline. The control group will be provided this information at the end of the RCT (12 weeks). The schedule of enrolment, interventions and assessments for the study is shown in table 1 . We will allow 3 weeks (lead-in phase) for all baseline biochemistry and AAC results to

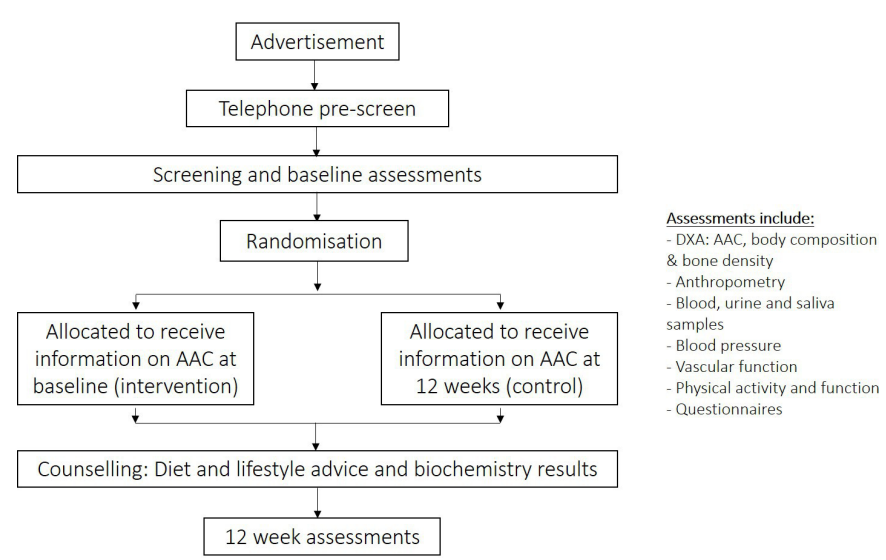

Figure 1 Study flow chart.

be available, before participants can be randomised and attend the counselling visit. The lead-in phase will include the following baseline assessments: (1) DXA scan for AAC assessment as well as bone density (hip and spine) and body composition; (2) anthropometry (body weight, height, waist and hip circumference); (3) a fasting blood sample for measurement of carotenoids (markers of FV intake), glucose, creatinine (to estimate eGFR) and lipids (cholesterol, triglycerides, high-density lipoprotein (HDL) cholesterol); (4) urine sample for urinary sodium, potassium and creatinine levels; (5) saliva test for nitrate and nitrite concentrations; (6) clinic BP; (7) arterial stiffness using pulse wave analysis (PWA); (8) physical activity assessed using ACTiGraph accelerometers and (9) physical function assessments (handgrip strength (GS), timed-up-and-go and gait speed). Questionnaires will be used to capture information on other health and lifestyle measures.

\section{Baseline counselling visit}

After being randomised to either the control or the intervention group, all participants will receive a 30-minute counselling session visit (week 0 ), as outlined in table 2. An unblinded counsellor trained in the need for impartiality will conduct all counselling sessions. To standardise counselling, all participants will watch three educational videos ( 1, 3 and $7 \mathrm{~min}$ each, respectively), will receive a booklet with dietary and lifestyle information and will be provided with their baseline biochemistry (CVD risk factor) results. This visit will run as follows:

1. The first video will contain information on the burden of CVD in Australia (from the Heart Foundation Australia). This will be immediately followed by a second video containing information on blood vessel disease (atherosclerosis) and how this leads to CVD. For the intervention group (AAC assessed), this video will also include a short explanation of AAC and its link to risk of heart attack and stroke. The video will conclude by encouraging dietary and physical activity change to reduce the risk of a heart attack or stroke.

2. Participants will be instructed to open an envelope containing (1) results from their baseline risk factor 
Table 1 Study design

\begin{tabular}{|c|c|c|c|c|}
\hline & Enrolment & Allocation & & \\
\hline Timepoint & Prescreen & Screening & Baseline & 12 weeks \\
\hline \multicolumn{5}{|l|}{ Enrolment } \\
\hline Informed consent & & $x$ & & \\
\hline Randomisation/allocation & & & $x$ & \\
\hline Intervention: Information on AAC & & & $x$ & \\
\hline Control: Information on AAC & & & & $x$ \\
\hline Dietary and lifestyle advice & & & $x$ & $x$ \\
\hline \multicolumn{5}{|l|}{ Assessments } \\
\hline Anthropometry & & & $x$ & $x$ \\
\hline Blood pressure & & & $x$ & $x$ \\
\hline Arterial stiffness & & & $x$ & $x$ \\
\hline Physical activity & & & $x$ & $x$ \\
\hline Physical function & & & $x$ & $x$ \\
\hline Questionnaires & & & $\mathrm{x}$ & $\mathrm{x}$ \\
\hline
\end{tabular}

AAC, abdominal aortic calcification.

Table 2 Baseline counselling session chart flow

\begin{tabular}{|c|c|c|c|}
\hline Intervention group & Estimated time & Control group & Estimated time \\
\hline $\begin{array}{l}\text { Video 1: National Heart } \\
\text { Foundation of Australia }\end{array}$ & $1 \mathrm{~min}$ & $\begin{array}{l}\text { Video 1: National Heart } \\
\text { Foundation of Australia }\end{array}$ & $1 \mathrm{~min}$ \\
\hline $\begin{array}{l}\text { Letter with visual and pictorial } \\
\text { representation of their AAC scan } \\
\text { results } \\
\text { Biochemistry and blood pressure } \\
\text { results } \\
\text { Letter to the GP regarding the } \\
\text { study }\end{array}$ & $5 \mathrm{~min}$ & $\begin{array}{l}\text { Letter without } A A C \text { scan results } \\
\text { Biochemistry and blood } \\
\text { pressure results }\end{array}$ & $5 \min$ \\
\hline $\begin{array}{l}\text { Video 3: dietary and lifestyle } \\
\text { information }\end{array}$ & $7 \min$ & $\begin{array}{l}\text { Video 3: dietary and lifestyle } \\
\text { information }\end{array}$ & $7 \mathrm{~min}$ \\
\hline $\begin{array}{l}\text { Face-to-face conversation with } \\
\text { investigator }\end{array}$ & $5 \mathrm{~min}$ & $\begin{array}{l}\text { Face-to-face conversation with } \\
\text { investigator }\end{array}$ & $5 \min$ \\
\hline $\begin{array}{l}\text { Postcounselling health status } \\
\text { questionnaire }\end{array}$ & $3 \min$ & $\begin{array}{l}\text { Postcounselling health status } \\
\text { questionnaire }\end{array}$ & $3 \min$ \\
\hline Total time & $29.5 \min$ & Total time & $29 \min$ \\
\hline
\end{tabular}

Information in italic represent the differences between intervention and control groups

AAC, abdominal aortic calcification; GP, general practitioner. 
assessments (both groups), including glucose and lipid levels and blood pressure-any values outside the reference ranges will be flagged for general practitioner (GP) follow-up; and (2) for the intervention (AAC assessed) group only, the results of their AAC scan will include a graphic image with a pictorial representation of the degree of calcification present and they will also be provided with an explanatory results letter for their GPs. Participants in both groups will be encouraged to share all of their results with their GP.

3 . The third video will then provide dietary and lifestyle counselling, which will summarise usual care standard dietary and lifestyle advice, with recommendations based on the Australian Dietary Guidelines ${ }^{27}$ and the recommendations on physical activity for older Australians, ${ }^{28}$ outlining three specific goals to be achieved and maintained (1) to increase fresh fruit intake by at least one serve per day $(150 \mathrm{~g} /$ day $)$ and increase vegetable intake by at least one serve per day $(75 \mathrm{~g} /$ day); (2) to improve other aspects of diet such as limiting intakes of saturated and trans fats, salt, sugar and alcohol and increasing the intake of whole grains, seeds and nuts; and (3) to increase physical activity and reduce sitting time. There will also be information on other dietary and lifestyle recommendations including serving sizes, food groups and alcohol intake (standard drinks) based on the Australians Dietary Guidelines ${ }^{27}$ and The Heart Foundation Australia recommendations $^{29}$; and key websites that may assist participants to achieve desired changes. The video content will be compiled in a hard copy booklet with additional detail in relation to each of the three study goals, which will also be provided to participants.

4. All participants will be provided with frequently asked questions and will have a 5-minute face-to-face conversation with the investigator, who will answer any questions raised. Participants will be asked to answer a postcounselling questionnaire to assess perceived health status.

At 12 weeks (end of RCT), all measurements performed at baseline will be repeated. In addition, the counselling sessions will be provided to both groups with the intervention group receiving the control group's baseline counselling and vice versa (ie, participants in the control group will receive the information about their level of advanced blood vessel disease at 12 weeks). A personalised report with the individual results and 12-week change will be provided to all participants by mail within 3 months of the end of the RCT.

\section{Patient and public involvement}

The study involved consumer representatives $(n=2)$ in all aspects of the study design providing input to the processes and assessments of this research project. The Consumer and Community Health Research Network, through The Consumer and Community Involvement Coordinator (Mr Ben Horgan) based at Edith Cowan University, assisted our Involvement Strategy by supporting us to find the most appropriate consumers for this work. The consumers were women over the age of 65 years who have attended five meetings, including four visits to the Medical Research Foundation and one visit to the Bone Density Department, over the last 12 months, prior to the start of the study. The women contributed to the design of the study, the informational material (results letter, handouts and videos) which supported the intervention and gave feedback about the burden of the intervention from the patient's perspective (including clinical assessments and online questionnaires). The two women from the community involved in this research provided feedback on their own clinical results and contributed to the dissemination plan of the study by suggesting which information should be provided and how it should be presented (graphic representation of scan results, providing feedback on the wording of all documents and questionnaires provided). The consumers were provided with a AU $\$ 30$ gift card for each hour involved in the study. The information sheet for the GPs was developed with feedback from five GPs at two busy Perth Medical Clinics.

\section{Outcome measures}

\section{Primary outcome measure}

The primary outcome for this study will be circulating total serum carotenoid concentrations as biomarkers of FV intake.

\section{Secondary outcomes}

Secondary outcomes will affect changes on: (1) intake of $\mathrm{FV}$ assessed using a validated semiquantitative food frequency questionnaire (FFQ) developed by the Cancer Council of Victoria, Australia ${ }^{30}$; (2) adherence to other dietary recommendations including intake of wholegrains and saturated and trans fats, salt, sugar and alcohol assessed using the FFQ and other questionnaires; (3) physical activity (minutes of moderate to vigorous, number of steps per day, total minutes of physical activity, sitting time) assessed using accelerometers along with the Community Healthy Activities Model Program for Seniors (CHAMPS) questionnaire; (4) CVD risk factors (including lipids, blood glucose, blood pressure); (5) body weight and body composition (including total body and regional fat mass $(\mathrm{kg})$ and body fat percentage, visceral adiposity $(\mathrm{kg})$, lean mass $(\mathrm{kg})$ and bone mineral density of the hip and spine $\left.\left(\mathrm{g} / \mathrm{cm}^{2}\right)\right)$; (6) physical function (GS, timed-upand-go and gait speed); (7) health-related measures such as sleep, smoking habits and knowledge and perception of CVD; (8) oral nitrite and nitrate concentrations; (9) medication and healthcare use and (10) psychological measures (such as depression and anxiety disorders and QoL).

\section{Sample size}

The required sample size for this study is 200 participants ( $\mathrm{n}=100$ control group; $\mathrm{n}=100$ intervention group). We expect a withdrawal rate of approximately $10 \%$ at 12 weeks. Rolling recruitment of participants will continue 
until the sample size of 200 is reached, which is estimated to take approximately 11 months. This sample will provide $85 \%$ power, with a two-sided type 1 error rate of alpha $=0.05$, to detect a $0.43 \mathrm{mg} / \mathrm{L}(\sim 25 \%)$ difference between control and intervention in total circulating carotenoids at 12 weeks. This is based on a conservative estimate of a SD of $1.0 \mathrm{mg} / \mathrm{L} .{ }^{32}$ In addition, a sample size of 100 participants in the intervention group will also provide more than $80 \%$ power to detect a dose-response effect (related to severity of AAC).

\section{Participant allocation}

Participants will be randomly allocated to either the intervention or control group in a 1:1 ratio. 'Based on previous studies, ${ }^{33} 34$ we developed the inclusion criteria in order to have at least $50 \%$ per group with evidence of AAC'. The sequence of allocation will be generated using Stata V.14 (StataCorp) and the user-written module called 'ralloc'. ${ }^{35}$ Randomised blocks of size 4 will be created, so that the randomisation is balanced at the completion of assignment for each block. The randomisation will also be stratified according to the age group (above or below 70 years) and sex. Within each strata, a sequential treatment code will be printed on paper in the order of treatment assignments. When the next available subject has completed baseline assessments, an investigator who is not involved in performing the intervention, data collection or data analysis will check the first available randomisation code in the sequence, reveal the treatment to be assigned (intervention or control) and assign an appropriate code (A or B) next to the participants' name on a list of participants. Both the participant and the investigators responsible for the counselling session will be unblinded throughout the trial. However, the researchers performing the outcome assessments and data analysis will be blinded to the treatments received until the end of data analysis when the treatment codes will be revealed.

\section{Data collection, management and analysis}

Study investigators will have access to the data collection forms and protocols using a secure shared drive. Participant information will be kept confidential with only the study investigators having access to the data. Data will be stored in password-protected databases accessible only by study investigators. Original backup files will be kept in a separate electronic folder. The research will be conducted within a research facility with electronic and keycode access. All forms, protocols and questionnaires used will be compiled in files, for easy accessibility. Checklists for clinic preparation and clinic days will be used to ensure consistency. The following clinic assessments will be performed.

\section{DXA scans and AAC scoring}

DXA scans will be performed at Gairdner Bone Densitometry Services, Sir Charles Gairdner Hospital, at the Perth site, using the latest fan beam technology (Hologic Horizon A densitometer). At the Deakin University site,
DXA scans will be performed using the Lunar iDXA, GE Lunar, Madison, USA. The lateral spine image captured will allow for assessment of AAC. Bone density of the hip and spine as well as a whole body scans for body composition assessment will also be performed at the time of AAC measurement. Focal AAC will be scored using a semiquantitative scoring system (scored 0-24; AAC24). This involves assessing the linear length of the vascular calcification relative to the L1-L4 lumbar vertebra. ${ }^{34} 36-39$ Briefly, the length of the calcifications on the anterior and posterior aortic walls is divided into four segments corresponding to the lumbar vertebrae $1-4$. For both the anterior and posterior aortic walls, aortic calcification is scored 0 if there is no calcification present, 1 if $\leq$ one-third of the aortic wall is calcified, 2 if $>$ one-third to $\leq$ two-thirds is calcified or 3 if $>$ two-thirds is calcified. As such, calcification corresponding to each of the four lumbar vertebra can achieve a maximum of $2 \times 3=6$ points with a combined maximum score of 24 points for L1-L4. All AAC scans will be analysed by experts in densitometric imaging (John Schousboe, University of Minnesota and/or Pawel Szulc, University of Lyon-France). The actual participant's scan and pictorial representation of the presence and extent of the AAC will be provided to the intervention group at baseline and the control group at 12 weeks. The whole-body DXA scan will be used to measure fat mass, lean mass and visceral adipose tissue (VAT) mass. A technique for estimating VAT using DXA involves selecting an area of interest with the superior border at the cranial border of L2 and the inferior border at the caudal border of L4. This has been shown to be well correlated with CT-derived and MRI-derived measures of VAT $\left(r^{2}>0.9\right){ }^{40}$

\section{Anthropometry}

Body weight will be measured using digital scales to the nearest $0.1 \mathrm{~kg}$ with participants wearing lightweight clothes and not wearing shoes. Height will be measured using a wall-mounted stadiometer to the nearest $0.1 \mathrm{~cm}$ while participants are not wearing shoes. BMI $\left(\mathrm{kg} / \mathrm{m}^{2}\right)$ will then be calculated as weight in kilogram, divided by height in metres squared. Waist circumference $(\mathrm{cm})$ will be measured using a Cescorf steel measuring tape, in the standing position at the narrowest area between the lateral lower rib and the iliac crest. ${ }^{41}$ Hip measurement $(\mathrm{cm})$ will be taken at the largest circumference of the lower abdomen. Waist and hip circumferences will be measured three times and the median of each reported as the score.

\section{Human specimens (blood, urine and saliva)}

Blood samples will be collected and standard biochemical analyses of fasting serum total cholesterol, HDLcholesterol and triglycerides will be performed. Low-density lipoprotein cholesterol concentrations will be calculated using the Friedewald formula. ${ }^{42}$ Glucose and creatinine (eGFR) levels will be used to screen for presence of diabetes and determine kidney function. Estimated FV intake (primary outcome) will be assessed 
using circulating serum carotenoid concentrations. Highperformance liquid chromatography methodology will be used to determine serum carotenoid concentrations of $\beta$-carotene, lycopene, $\alpha$-carotene, $\beta$-cryptoxanthin, lutein and zeaxanthin, as previously described. ${ }^{32}{ }^{43}$ Serum carotenoids will be measured to assess FV intake, as FV are the primary contributors to intake of carotenoids (such as $\alpha$ carotene, $\beta$-carotene and lutein/zeaxanthin $)^{44}$ and they are recognised and commonly used biomarkers of $\mathrm{FV}$ consumption. ${ }^{45}$ A 24-hour urine sample will be collected in a sterile container provided. Urine samples will be analysed for electrolytes including creatinine, sodium and potassium. Saliva samples will be obtained to assess salivary nitrate and nitrite concentrations and to determine the nitrate to nitrite reduction ratio. ${ }^{46}$ Participants will be asked to sit quietly for $5 \mathrm{~min}$ while spitting into a sterile specimen container. Saliva aliquots will be stored at $-80^{\circ} \mathrm{C}$.

\section{Clinic BP}

Clinic BP will be assessed using a validated automatic BP monitor. After participants have rested for $5 \mathrm{~min}$ in a supine position, BP measurements will be assessed on five occasions at $1 \mathrm{~min}$ intervals. The first measurement will be discarded and the mean of the final four readings will be calculated.

\section{Pulse wave analysis}

PWA will be used to assess central aortic blood pressure and stiffness (augmentation index, \%), ${ }^{47}$ using the Sphygmocor Xcel device as described previously. ${ }^{48}$

\section{Actigraphy}

Accelerometry (ActiGraph GT3X, Pensacola, Florida, USA) will be used at baseline (lead-in-phase) and 12 weeks, each for 7 consecutive days to investigate average daily physical activity levels. Of note, the ActiGraph device is the most widely used accelerometer in physical activity research. ${ }^{49}$ Briefly, participants will be instructed to wear the accelerometer over the right hip secured with an elastic band while awake and during sleep (if possible) and to remove the accelerometer when doing water activities. Accelerometers are ideal for determining physical activity as they require minimal input from the participant over the collection period. To improve the accuracy of our data, participants will also be asked to report any times the monitor is removed using a concurrent daily Activity Monitor Record/Nap Time Questionnaire during the 7-day period. Physical activity outcome measures will include moderate-to-vigorous physical activity ( $\mathrm{min} /$ day), total physical activity (min/day), sedentary time (min/ day) and daily step count (steps/day). These measures will be quantified using the ActiLife Data Analysis Software provided by the manufacturer (ActiLife V.6.13.4, Pensacola, Florida, USA).

\section{Muscle strength and physical function}

Muscle strength and physical function will be assessed by three tests: (1) GS of both the dominant and non-dominant hand will be recorded as the highest of three attempts ${ }^{50}$ using a handheld dynamometer (Jamar Hydraulic Hand Grip Dynamometer; Nottinghamshire, UK); (2) timed up-and-go will be used to assess mobility, in which participants are timed (using a stopwatch) raising from a seated position on a standard arm chair $(45 \mathrm{~cm})$ and asked to walk at a comfortable and safe pace to a $3 \mathrm{~m}$ mark, turning to return to their seated position on the chair with their back touching the backrest of the chair. ${ }^{51}$ Participants will be instructed to perform one practice trial before the next two recorded attempts with the fastest time reported and (3) gait speed in which participants will be timed while walking at their usual pace over $4 \mathrm{~m}$. Participants will be instructed to walk at their usual pace over $8 \mathrm{~m}$ and the time taken to walk the middle $4 \mathrm{~m}$ will be recorded on a stopwatch to determine gait speed. Time taken to walk the first and last $2 \mathrm{~m}$ of the course is excluded due to the acceleration and deceleration phases, respectively. ${ }^{52}$

\section{Questionnaires}

Dietary intake will be assessed using a semiquantitative FFQ developed by the Cancer Council of Victoria, Australia, ${ }^{31}$ administered by one of the study investigators at each clinic visit. A link for a series of questionnaires to be completed online, which have been used widely in clinical research including by our team, will be sent to participants at baseline and 12 weeks, as described below: (1) Demographic and Lifestyle Questionnaire: will be used to assess demographic and lifestyle information, smoking habits and knowledge and perception of CVD risk; (2) Beverages Questionnaire: a self-administered beverage questionnaire will be used to estimate the average daily or weekly intake of beverages across 12 types of drinks (tea, herbal tea, coffee, decaffeinated coffee, chocolate, fruit juice, soft drinks, diet drinks, dairy milk, non-dairy milk, fruit and/or vegetable smoothies and water) as well as alcohol intake (beer, red and white wine and spirits); (3) CHAMPS questionnaire: a self-reported measure of physical activity to estimate weekly (4 weeks timeframe) frequency of participation (types) and energy expenditure (intensity levels) of physical activities in older adults $^{53}$; (4) Longitudinal Ageing Study Amsterdam questionnaire will be used to assess sedentary behaviour ${ }^{54}$; (5) well-being and QoL will be assessed by the Medical Outcome Study Short Form-36 (SF-36 $)^{55}$ and the Suboptimal Health Status questionnaire (SHSQ-25) status. ${ }^{56}$ SF36 includes eight domains of physical functioning, role-physical, bodily pain, general health, vitality, social functioning, role-emotional and mental health, which are combined to form two key domains-physical functioning and mental health. SHSQ-25 is composed of 25 items and includes five subscales: fatigue, the cardiovascular system, the digestive tract, the immune system and mental status; (6) Depression Anxiety Stress Scales-21: is a self-report questionnaire designed to measure the negative sensitive conditions of depression, anxiety and stress; (7) Bowel Symptoms Questionnaire will be used to investigate bowel habits along with the Bristol stool 
chart, a medical tool designed to assess stool consistency (types 1 and 2 indicate constipation, types 3 and 4 are the 'ideal stools' and types 5-7 suggest diarrhoea or bowel urgency $)^{57}$; (8) Readiness to Change and Wellbeing Questionnaire is a behaviour modification questionnaire (developed by the WorkStrong program, University of California system wide program) which focuses on individuals' readiness to change over seven different aspects: life satisfaction, energy, weight, exercise, nutrition, health and stress and mental health; (9) Pittsburgh Sleep Quality Index: a questionnaire used to measure the quality and patterns of sleep in older adults ${ }^{58}$; (10) current medication use: a comprehensive list of medications (which will be cross-checked with bottles/packets of tablets concomitantly) and; (11) Health Care Use Questionnaire: will be used to investigate any procedures or further medical tests requested by GPs as well as visits to healthcare professionals. Online questionnaires will be checked for completeness prior to each clinic visit and any missing questions will be completed at the clinic visit. The 'Life's simple 7' metric from the American Heart Association will be used to assess participant's 'ideal' cardiovascular health. This considers seven risk factors that people can improve through lifestyle changes: quit smoking, increase their physical activity, achieve a healthy weight, eat a healthy diet, reduce their blood glucose, control their cholesterol and manage their blood pressure. ${ }^{59}$

\section{Other forms}

Participants will be asked to complete a 24-hour urine record form along with the Flavonoids questionnaire, which will be used to estimate the consumption of foods and beverages rich in flavan-3-ols in the 24 hours prior to their visit. The number of serves consumed $(0,1,2,3$ or $4+)$ across 22 foods and beverages will be assessed.

A postcounselling health status questionnaire will be used to assess the participants' perceptions and intentions towards CVD risk and to the study proposed changes, immediately after receiving information on CVD and diet and lifestyle (counselling visit). The questions aim to explore participants perceived susceptibility and severity ${ }^{6061}$ in relation to the heart disease video content. It also explores the response efficacy, selfefficacy and their intentions (Likert scale: 1=strongly disagree; $5=$ strongly agree) towards the three main study goals.

\section{Data management}

The majority of data will be electronic and directly sent to the Qualtrics database. Online questionnaires have been designed with mandatory answers required to avoid missing data. Nevertheless, these data will be checked by an investigator prior to clinic day. Data from clinic assessments and paper questionnaires will be entered into individual spreadsheets by a study investigator. A representative number of files will be selected by a different investigator, who will check whether the information matches that in the electronic spreadsheets.

\section{Statistical methods}

Statistical analysis will be performed using IBM SPSS (V.25; IBM) ${ }^{62}$ and Stata V.14 (StataCorp). Descriptive statistics will be used to describe the individuals characteristics at baseline. The primary analysis will be performed on a modified intention-to-treat basis with the population defined as those randomised participants for whom there are baseline measurements. Data will be assessed for outliers and normality prior to analysis and log-transformed if necessary. Baseline and 12-week values for outcome measures and between-group differences will be presented as means and SDs. The treatment effect for each of the outcomes will be analysed using generalised linear mixed models to examine the baseline adjusted differences between intervention and control in 12 -week values. In addition to including visit and treatment group as fixed effects in each model, an interaction term between visit and treatment groups will be included and used to determine the effect of the intervention. The randomisation site and the individual will both be included in the models as random effects to account for clustering. A per-protocol analysis will be performed as a secondary analysis using only those participants with measures recorded at baseline and 12 weeks. An overall two-sided type- 1 error rate of $\mathrm{p}<0.05$ will be used to assess statistical significance for all hypothesis testing.

\section{Monitoring}

Participants will be monitored for any adverse events during clinical assessments by the study investigators which have been detailed in the participant information form. The study clinical coordinator will oversee all aspects of participant management where required.

\section{ETHICS AND DISSEMINATION}

Ethics approval for this study has been granted by the Edith Cowan University Human Research Ethics Committee (HREC) (Project number: 20513 HODGSON) and Deakin University HREC (Project number: 2019-220). Results of this study will be published in peer-reviewed academic journals and presented in scientific meetings and conferences. Information regarding consent, confidentiality, access to data, ancillary and post-trial care and dissemination policy have been disclosed in the participant information form.

\section{Author affiliations}

${ }^{1}$ School of Medical and Health Sciences, Edith Cowan University, Joondalup, Western Australia, Australia

${ }^{2}$ Medical School, University of Western Australia, Perth, Western Australia, Australia ${ }^{3}$ Park Nicollet Osteoporosis Center and Health Partners Institute, and Division of Health Policy and Management, University of Minnesota, Minneapolis, Minnesota, USA

${ }^{4}$ Flinders Centre for Epidemiology and Biostatistics, Flinders University, Adelaide, South Australia, Australia

${ }^{5}$ Department of Endocrinology and Diabetes, Sir Charles Gairdner Hospital, Nedlands, Western Australia, Australia

${ }^{6}$ INSERM UMR 1033, University of Lyon, Hospices Civils de Lyon, Lyon, France ${ }^{7}$ Faculty of Science, School of Human Sciences, University of Western Australia, Perth, Western Australia, Australia 
${ }^{8}$ Department of Psychology, College of Healthcare Sciences, James Cook University, Townsville, Queensland, Australia

${ }^{9} \mathrm{Hinda}$ and Arthur Marcus Institute for Aging Research, Hebrew SeniorLife, Beth Israel Deaconess Medical Center, Harvard Medical School, Boston, Massachusetts, USA

${ }^{10}$ Department of Renal Medicine, Sir Charles Gairdner Hospital, Nedlands, Western Australia, Australia

${ }^{11}$ Department of Cardiology, Sir Charles Gairdner Hospital, Perth, Western Australia, Australia

${ }^{12}$ Institute for Physical Activity and Nutrition, School of Exercise and Nutrition

Science, Deakin University, Melbourne, VIC, Australia

${ }^{13}$ Centre for Kidney Research, Children's Hospital at Westmead, School of Public Health, Sydney Medical School, The University of Sydney, Sydney, NSW, Australia

Twitter Mandy Stanley @stanley_mandy and Amanda Devine @AdMandydevine

Acknowledgements The investigators thank the organisations and individuals who helped develop, improve and publicise this research project protocol, in particular Mr Ben Horgan, the Consumer and Community Involvement Coordinator from The Consumer and Community Health Research Network (CCHRN), based at Edith Cowan University, and the community representatives (Mrs Barbara Horgan and Mrs. Kathleen Joy Foyle) involved in this research project. The investigators would also like to thank Professor Moira Sim who helped to develop and evaluate the information sheet for general practitioners for the MODEL study.

Contributors JMH, JRL and RD contributed to the development of the study concept. SR-B, CPB, MS, LB, RA, EC, NPB, JTS, RW, KZ, PS, BJ, JD, MPS, KLC, DPK, WHL, MPS, AD, PLT, JG, BDR, RD, JRL and JMH provided input on the study design and eligibility criteria for the study. SR-B, CPB, MS, LB, BJ, JD, MPS, RD, JRL and JMH developed the script for both videos on diet and lifestyle recommendations and the handout. CPB created the video on diet and lifestyle and the booklet for the counselling session. NPB assisted with the methodology of the clinical trial. EC and MS voiced over the video on diet and lifestyle. All authors assisted in further development and implementation of the protocol. SR-B, CPB, MS, LB, RA, EC, JG and BDR are involved in the acquisition of data. JTS, PS and JRL had input in the development of the graphic images of the AAC results. RW created the randomisation codes and had input in the statistical analysis plan. $\mathrm{KZ}$ is responsible for the bone density scans. MPS, DPK, WHL and PLT provided clinical input. BJ and JD contributed to the development and evaluation of the instruments to capture behaviour change and individual's wellbeing. MPS voiced over the video on atherosclerosis and is the clinician responsible for checking the clinical/medical tests of the study. KLC helped to develop the information on physical activity and to choose the instruments to measure physical activity and the physical function tests. $A D$ assisted with the dietary aspects of the study. SRB, JRL and JMH contributed to drafting of the protocol and provided approval for the version submitted for publishing. JRL, JMH and CPB are supervisors for the PhD candidate (SR-B). SR-B, CPB, MS, LB, RA, EC, NPB, JTS, RW, KZ, PS, BJ, JD, MPS, KLC, DPK, WHL, MPS, AD, $P L T, J G, B D R, R D, J R L$ and JMH contributed and approved the final manuscript.

Funding This research received no specific grant from any funding agency in the public, commercial or not-for-profit sectors'. The salary of JRL is supported by a National Heart Foundation of Australia future leader fellowship (ID 102817). DPK's time was supported by a grant from the National Institute of Arthritis, Musculoskeletal and Skin Diseases (R01 AR 41398). The salary of JMH is supported by a National Health and Medical Research Council of Australia Senior Research Fellowship (ID 1116973). The salary of LCB is supported by an NHMRC of Australia Emerging Leadership Investigator Grant (ID: 1172987) and a National Heart Foundation of Australia Post-Doctoral Research Fellowship (ID: 102498).

Competing interests None declared.

Patient consent for publication Not required.

Provenance and peer review Not commissioned; externally peer reviewed.

Open access This is an open access article distributed in accordance with the Creative Commons Attribution Non Commercial (CC BY-NC 4.0) license, which permits others to distribute, remix, adapt, build upon this work non-commercially, and license their derivative works on different terms, provided the original work is properly cited, appropriate credit is given, any changes made indicated, and the use is non-commercial. See: http://creativecommons.org/licenses/by-nc/4.0/.

\section{ORCID iDs}

Simone Radavelli-Bagatini http://orcid.org/0000-0001-6821-5217

Marc Sim http://orcid.org/0000-0001-5166-0605
Mandy Stanley http://orcid.org/0000-0002-7958-5181

\section{REFERENCES}

1 WHO. Cardiovascular diseases (CVDs). In: Organization WWH, ed. Who fact sheets. WHO, 2017.

2 AlHW. Cardiovascular disease snapshot. Australian Institute of Health and Welfare, 2018.

3 Mozaffarian D, Benjamin EJ, AS G, et al. Heart disease and stroke statistics-2016 update a report from the American heart association. Circulation 2016;133:e38-48.

4 WHO. Diet, nutrition, and the prevention of chronic diseases: report of a joint WHO/FAO expert consultation (Report No.: 924120916X). World Health Organization, 2003.

5 Artinian NT, Fletcher GF, Mozaffarian D, et al. Interventions to promote physical activity and dietary lifestyle changes for cardiovascular risk factor reduction in adults: a scientific statement from the American heart association. Circulation 2010;122:406-41.

6 Wang $X$, Ouyang Y, Liu J, et al. Fruit and vegetable consumption and mortality from all causes, cardiovascular disease, and cancer: systematic review and dose-response meta-analysis of prospective cohort studies. BMJ 2014;349:g4490.

7 Ezzati M, Riboli E. Behavioral and dietary risk factors for noncommunicable diseases. N Engl J Med 2013;369:954-64.

8 Mozaffarian D, Capewell S. United nations' dietary policies to prevent cardiovascular disease. BMJ 2011;343:d5747.

9 Aune D, Giovannucci E, Boffetta P, et al. Fruit and vegetable intake and the risk of cardiovascular disease, total cancer and all-cause mortality - a systematic review and dose-response meta-analysis of prospective studies. Int J Epidemiol 2017;46:1029-56.

10 Lee-Kwan SH, Moore LV, Blanck HM, et al. Disparities in StateSpecific Adult Fruit and Vegetable Consumption - United States, 2015. MMWR Morb Mortal Wkly Rep 2017;66:1241-7.

11 ABS. Australian Health Ministers, 2009. 'Fourth National Mental Health Plan - an agenda for collaborative government action in mental health 2009-2014'. Australian Bureau of Statistics, Commonwealth of Australia, 2015.

12 ABS. 4324.0.55.001 - Microdata: National Health Survey, 2014-15. Canberra: ABS AIHW analysis of Basic Confidentialised Unit Record File, 2016.

13 Cadilhac DA, Magnus A, Sheppard L, et al. The societal benefits of reducing six behavioural risk factors: an economic modelling study from Australia. BMC Public Health 2011;11:483.

14 Deloitte Access Economics. The impact of increasing vegetable consumption on health expenditure. horticulture innovation Australia, 2016.

15 Blaha MJ, Silverman MG, Budoff MJ. Is there a role for coronary artery calcium scoring for management of asymptomatic patients at risk for coronary artery disease?: clinical risk scores are not sufficient to define primary prevention treatment strategies among asymptomatic patients. Circ Cardiovasc Imaging 2014;7:398-408.

16 McClelland RL, Jorgensen NW, Budoff M, et al. 10-Year coronary heart disease risk prediction using coronary artery calcium and traditional risk factors: derivation in the MESA (multi-ethnic study of atherosclerosis) with validation in the HNR (heinz Nixdorf recall) study and the DHS (Dallas heart study). J Am Coll Cardiol 2015;66:1643-53.

17 Criqui MH, Denenberg JO, McClelland RL, et al. Abdominal aortic calcium, coronary artery calcium, and cardiovascular morbidity and mortality in the multi-ethnic study of atherosclerosis. Arterioscler Thromb Vasc Biol 2014;34:1574-9.

18 Schousboe JT, Richter SA, Beran MS. Potential clinical impact of abdominal aortic calcification on bone density lateral spine images. $J$ Clin Densitom 2016;19:436-43.

19 McEvoy JW, Blaha MJ, Defilippis AP, et al. Coronary artery calcium progression: an important clinical measurement? A review of published reports. J Am Coll Cardiol 2010;56:1613-22.

20 Rozanski A, Gransar H, Shaw LJ, et al. Impact of coronary artery calcium scanning on coronary risk factors and downstream testing the EISNER (early identification of subclinical atherosclerosis by noninvasive imaging research) prospective randomized trial. J Am Coll Cardiol 2011;57:1622-32.

21 Mamudu HM, Paul TK, Veeranki SP, et al. The effects of coronary artery calcium screening on behavioral modification, risk perception, and medication adherence among asymptomatic adults: a systematic review. Atherosclerosis 2014;236:338-50.

22 Rosenstock IM. The health belief model and preventive health behavior. Health Educ Monogr 1974;2:354-86.

23 Armitage CJ, Conner M. Social cognition models and health behaviour: a structured review. Psychol Health 2000;15:173-89. 
24 Maloney EK, Lapinski MK, Witte K. Fear appeals and persuasion: a review and update of the extended parallel process model. Soc Personal Psychol Compass 2011;5:206-19.

25 Chan A-W, Tetzlaff JM, Altman DG, et al. Spirit 2013 statement: defining standard protocol items for clinical trials. Ann Intern Med 2013;158:200-7.

26 Bredy C, Ministeri M, Kempny A, et al. New York heart association (NYHA) classification in adults with congenital heart disease: relation to objective measures of exercise and outcome. Eur Heart J Qual Care Clin Outcomes 2018;4:51-8.

27 National Health and Medical Research Council. Australian dietary guidelines. Canberra: National Health and Medical Research Council, 2013.

28 The Department of Health: Australian Government. Recommendations on physical activity for health for older Australians, 2013. Available: http://www.health.gov.au/internet/main/ publishing.nsf/content/phd-physical-rec-older-guidelines

29 The Heart Foundation. Heart healthy eating principles Australia, 2017. Available: https://www.heartfoundation.org.au/forprofessionals/food-and-nutrition/heart-healthy-eating-principles

30 Hodge A, Patterson AJ, Brown WJ, et al. The anti cancer Council of Victoria FFQ: relative validity of nutrient intakes compared with weighed food records in young to middle-aged women in a study of iron supplementation. Aust N Z J Public Health 2000;24:576-83.

31 Ireland P, Jolley D, Giles G, et al. Development of the Melbourne FFQ: a food frequency questionnaire for use in an Australian prospective study involving an ethnically diverse cohort. Asia Pac J Clin Nutr 1994;3:19-31.

32 Blekkenhorst LC, Lewis JR, Prince RL, et al. Nitrate-rich vegetables do not lower blood pressure in individuals with mildly elevated blood pressure: a 4-wk randomized controlled crossover trial. Am J Clin Nutr 2018;107:894-908.

33 Lewis JR, Schousboe JT, Lim WH, et al. Long-Term atherosclerotic vascular disease risk and prognosis in elderly women with abdominal aortic calcification on lateral spine images captured during bone density testing: a prospective study. J Bone Miner Res 2018;33:1001-10.

34 Schousboe JT, Lewis JR, Kiel DP. Abdominal aortic calcification on dual-energy X-ray absorptiometry: methods of assessment and clinical significance. Bone 2017;104:91-100.

35 Ryan P. Australia S. sxd1. 2 random allocation of treatments balanced in blocks: update. Stata March 2000 technical STB-54.49.

36 Lewis JR, Schousboe JT, Lim WH, et al. Abdominal aortic calcification identified on lateral spine images from bone densitometers are a marker of generalized atherosclerosis in elderly women. Arterioscler Thromb Vasc Biol 2016;36:166-73.

37 Bondonno N, Lewis J, Prince R, et al. Fruit intake and abdominal aortic calcification in elderly women: a prospective cohort study. Nutrients 2016;8:159. a.

38 Schousboe JT, Taylor BC, Kiel DP, et al. Abdominal aortic calcification detected on lateral spine images from a bone densitometer predicts incident myocardial infarction or stroke in older women. Journal of Bone and Mineral Research 2008;23:409-16.

39 Schousboe JT, Claflin D, Barrett-Connor E. Association of coronary aortic calcium with abdominal aortic calcium detected on lateral dual energy X-ray absorptiometry spine images. Am J Cardiol 2009:104:299-304.

40 Hill AM, LaForgia J, Coates AM, et al. Estimating abdominal adipose tissue with DXA and anthropometry. Obesity 2007;15:504.

41 Marfell-Jones MO, Carter JL, Stewart A. International standards for anthropometric assessment. Potchefstroom, South Africa: The
International Society for the Advancement of Kinanthropometry, 2007: 131.

42 Friedewald WT, Levy RI, Fredrickson DS. Estimation of the concentration of low-density lipoprotein cholesterol in plasma, without use of the preparative ultracentrifuge. Clin Chem 1972;18:499-502.

43 Wood LG, Garg ML, Smart JM, et al. Manipulating antioxidant intake in asthma: a randomized controlled trial. Am J Clin Nutr 2012;96:534-43.

44 Block G, Norkus E, Hudes M, et al. Which plasma antioxidants are most related to fruit and vegetable consumption? Am J Epidemiol 2001;154:1113-8.

45 Andersen LF, Veierød MB, Johansson L, et al. Evaluation of three dietary assessment methods and serum biomarkers as measures of fruit and vegetable intake, using the method of triads. $\mathrm{Br} J$ Nutr 2005;93:519-27.

46 Bondonno CP, Croft KD, Puddey IB, et al. Nitrate causes a dosedependent augmentation of nitric oxide status in healthy women. Food Funct 2012;3:522-7.

47 Stoner L, Young JM, Fryer S. Assessments of arterial stiffness and endothelial function using pulse wave analysis. Int $J$ Vasc Med 2012;2012:1-9.

$48 \mathrm{H}$ wang $\mathrm{M}-\mathrm{H}$, Yoo J-K, Kim H-K, et al. Validity and reliability of aortic pulse wave velocity and augmentation index determined by the new cuff-based SphygmoCor Xcel. J Hum Hypertens 2014;28:475-81.

49 Bassett DR, Troiano RP, McClain JJ, et al. Accelerometer-based physical activity: total volume per day and standardized measures. Med Sci Sports Exerc 2015;47:833-8.

50 Kallman DA, Plato CC, Tobin JD. The role of muscle loss in the age-related decline of grip strength: cross-sectional and longitudinal perspectives. J Gerontol 1990;45:M82-8.

51 Podsiadlo D, Richardson S. The Timed "Up \& Go": A Test of Basic Functional Mobility for Frail Elderly Persons. J Am Geriatr Soc 1991;39:142-8.

52 Bisca GW, Fava LR, Morita AA, et al. 4-Meter gait speed test in chronic obstructive pulmonary disease: interrater reliability using a STOPWATCH. J Cardiopulm Rehabil Prev 2018;38:E10-13.

53 Stewart AL, Mills KM, King AC, et al. Champs physical activity questionnaire for older adults: outcomes for interventions. Med Sci Sports Exerc 2001;33:1126-41.

54 Adam TC, Epel ES, Stress EES. Stress, eating and the reward system. Physiol Behav 2007:91:449-58.

55 Ware JE, Kosinski M, Keller SD. The SF-36 health survey, 1990.

56 Yan Y-X, Liu Y-Q, Li M, et al. Development and evaluation of a questionnaire for measuring suboptimal health status in urban Chinese. J Epidemiol 2009;19:333-41.

57 Lewis SJ, Heaton KW. Stool form scale as a useful guide to intestina transit time. Scand J Gastroenterol 1997;32:920-4

58 Buysse DJ, Reynolds CF, Monk TH, et al. The Pittsburgh sleep quality index: a new instrument for psychiatric practice and research. Psychiatry Res 1989;28:193-213.

59 Lloyd-Jones DM, Hong Y, Labarthe D, et al. Defining and setting national goals for cardiovascular health promotion and disease reduction: the American heart association's strategic impact goal through 2020 and beyond. Circulation 2010;121:586-613.

60 Witte K. Putting the fear back into fear appeals: the extended parallel process model. Commun Monogr 1992;59:329-49.

61 Witte K, Allen M. A meta-analysis of fear appeals: implications for effective public health campaigns. Health Educ Behav 2000;27:591-615.

$62 \mathrm{lbm}$ C. Ibm SPSS statistics for windows, version Q3 25.0. Armonk, NY: IBM Corp, 2017 